\title{
Qualitätsmessung und qualitätsorientierte Vergütung aus Sicht des GKV-Spitzenverbandes
}

W.-D. Leber

Qualitätsmanagement

Schlüsselwörter

Qualitätsmessung

Qualitätsindikatoren

- Gemeinsamer

Bundesausschuss

- Sektorübergreifende Qualitätssicherung

Qualitätssicherung mit Routinedaten

Selektiv-Verträge

\section{Keywords}

measurement of quality

quality indicators

Gemeinsamer Bundesausschuss (Federal joint Committee)

$\checkmark$ quality assurance with administrative data

selective contracts
Institut

GKV-Spitzenverband, Berlin

Bibliografie

DOI $10.1055 / \mathrm{s}-0029-1242672$

Dtsch Med Wochenschr 2009;

134: S316 - (c) Georg Thieme

Verlag KG Stuttgart - New York .

ISSN 0012-0472

Korrespondenz

Dr. Wulf-Dietrich Leber

GKV-Spitzenverband

Mittelstr. 51

10117 Berlin

030/2062882200

eMail wulf-dietrich.leber@

gkv-spitzenverband.de
Versuche, Qualität und Vergütung medizinischer Leistungen zu definieren, finden sich bereits auf den ersten Gesetzestafeln der Menschheit (Codex Hammurapi, 1750 v. Chr.). Belebt wird die Debatte durch US-amerikanische Initiativen unter der Überschrift „pay for performance (P4P)“. Neu ist der qualitätsorientierte Ansatz für Deutschland nicht; in den DRG-Abrechnungsregeln finden sich bereits qualitätssichernde Steuerungsinstrumente, wie z.B. die Fallzusammenführung bei Komplikationen.

Gleichwohl existieren noch 2 Parallelwelten: die Welt der Vergütung und die Welt der Qualitätssicherung. Gesetzliche Bestimmungen (z.B. §299 Abs. 3 SGB V) verhindern bislang, dass Qualitätsdaten vergütungsrelevant werden. Hier besteht Handlungsbedarf.

Basis für jede qualitätsorientierte Vergütung ist Qualitätsmessung und Qualitätstransparenz. Die Suche nach geeigneten Messgrößen führt zu einem schwer überschaubaren Kosmos von QS-Indikatoren: rund $200 \mathrm{BQS}$ (Bundesgeschäftsstelle für Qualitätssicherung)-Indikatoren, mehre hundert HeliosIndikatoren, diverse QSR(Qualitätssicherung mit Routinedaten)-Tracer, über 100 QiSA(Qualitätsindikatorensystem für die ambulante Versorgung)Indikatoren für ambulante Versorgung, mehrere dutzend AQIK(Ambulante Qualitätsindikatoren und Kennzahlen)-Indikatoren (nach einer Vorauswahl aus rund 2000 international verfügbaren Indikatoren).

Die Suche nach dem idealen Indikator für qualitätsorientierte Vergütung ist schwierig. Der ideale Indikator ist qualitätsdifferenzierend, misst Ergebnisqualität, ist evidenzbasiert, signifikant und risikoadjustiert, ist aufwandsarm zu erheben (möglichst Routinedaten), liefert zeitnah Ergebnisse und ordnet medizinische Qualität verursachungsgerecht $\mathrm{zu}$.

Das zentrale Gremium zur Messung der medizinischen Qualität ist der Gemeinsame Bundesausschuss (G-BA). Der gesetzliche Auftrag, Qualität sektorübergreifend zu messen, wird erst zögerlich umgesetzt. Die wesentliche datentechnische Herausforderung ist die Mehrpunktmessung. Sie soll insbesondere sektorgleiche Qualitätssicherung und Verlaufsmessung sowie die Abbildung intersektoraler Prozesse ermöglichen. Der schnellste Weg zu sektorübergreifenden Qualitätssicherungsverfahren ist die Erweiterung bestehender BQS-Verfahren (sektorgleiche Anwendung, fallübergreifende Verlaufsmessung).
Eine ergänzende Nutzung von Kassendaten ist insbesondere für Mortalitätsdaten essenziell. Sie ist bisher nicht gesetzlich vorgesehen, aber im Rahmen selektiver Verträge möglich (vgl. das AOK-Helios-Projekt „Qualitätssicherung mit Routinedaten“).

Eine stärker wettbewerblich organisierte Versorgung mit Selektiv-Verträgen wird neue Herausforderungen für die Qualitätssicherung mit sich bringen. Aufgrund mangelnder Signifikanz bei geringen Fallzahlen könnten öffentlich verfügbare GKV-weite Qualitätsmessungen auch bei Selektiv-Verträgen eine bedeutende Rolle spielen. Möglicherweise gilt: „Kollektiv messen, selektiv kontrahieren."

Autorenerklärung: Der Autor erklärt, dass keine relevanten finanziellen Verbindungen in Bezug auf dieses Manuskript bestehen. 